\title{
Regulação emocional, satisfação sexual e comportamento sexual de risco em mulheres vítimas de abuso sexual na infância
}

\author{
Emotion regulation, sexual satisfaction and risky \\ sexual behavior in women victims of \\ sexual abuse in childhood
}

Cris Aline KRINDGES ${ }^{1}$ ID 0000-0003-2974-4567
Luísa Fernanda HABIGZANG ${ }^{1}$ iD 0000-0002-0262-0356

Resumo

Buscou-se investigar questões relacionadas à regulação emocional, à satisfação sexual e ao comportamento sexual de risco em mulheres adultas com histórico de abuso sexual na infância. Participaram desta pesquisa oito mulheres, com média de idade 38 anos, que responderam aos instrumentos de autorrelato sobre regulação emocional e satisfação sexual e a uma entrevista em profundidade, que avaliou aspectos do contexto familiar, emocional, relacionamentos amorosos e também sexuais, abordando questões sobre comportamento sexual de risco. O delineamento utilizado foi exploratório, descritivo com abordagem mista. Os resultados demonstraram que todas as participantes avaliadas possuíam dificuldades de regulação emocional, em menores ou maiores níveis. Em relação à satisfação sexual, seis das participantes obtiveram índices positivos de satisfação, e algumas delas relataram desejo e satisfação sexual preservados. No entanto, outras participantes relataram dificuldades, como aversão sexual e comportamento hipersexualizado. Comportamentos sexuais de risco foram identificados em duas das participantes e vinculados a comportamentos do passado.

Palavras-chave: Abuso sexual; Regulação emocional; Satisfação sexual; Sexo sem proteção.

\begin{abstract}
This research aimed to investigate aspects regarding emotion regulation, sexual satisfaction and risky sexual behavior in adult women with history of childhood sexual abuse. Eight women with an average age of 38 were subjects for this



1 Pontifícia Universidade Católica do Rio Grande do Sul, Programa de Pós-Graduação em Psicologia, Grupo de Pesquisa: Violência, Vulnerabilidade e Intervenções Clínicas. Av. Ipiranga, 6681, Prédio 11, $9^{\circ}$ andar, 90619-900, Porto Alegre, RS, Brasil. Correspondência para/Correspondence to: C.A. KRINDGES. E-mail: <cris.krindges@gmail.com>.

Artigo elaborado a partir da dissertação de C. A. KRINDGES, intitulada "Regulação emocional, satisfação sexual e comportamento sexual de risco em mulheres vítimas de abuso sexual na infância”. Pontifícia Universidade Católica do Rio Grande do Sul, 2016.

$\boldsymbol{\nabla} \mathbf{\nabla} \boldsymbol{\nabla}$

Como citar este artigo/How to cite this article

Krindges, C. A., \& Habigzang, L. F. (2018). Regulação emocional, satisfação sexual e comportamento sexual de risco em mulheres vítimas de abuso sexual na infância. Estudos de Psicologia (Campinas), 35(3), 321-332. http://dx.doi.org/10.1590/1982-02752018000300010
\end{abstract}


study. They answered to the self-report instruments about emotion regulation and sexual satisfaction, and to an in-depth interview which assessed aspects related to the family, emotional background, besides the loving and sexual relations, including aspects on risky sexual behavior. A descriptive, quantitative and qualitative outlining was carried out. The results showed that all the evaluated presented emotion dysregulation in major or minor levels. Regarding sexual satisfaction, six participants presented positive rates of satisfaction and some of them reported the preservation of there desire and sexual satisfaction. However, other participants reported some difficulties such as sexual aversion and hypersexual behavior. Some risky sexual behaviors were identified in two of these participants and linked to past behaviors.

Keywords: Sexual abuse; Emotional regulation; Sexual satisfaction; Unsafe sex.

O Abuso Sexual na Infância (ASI) pode ser compreendido a partir do envolvimento de crianças em interações de cunho sexual praticadas por pessoas em estágio psicossexual mais avançado do que a vítima (World Health Organization, 2002). O ASI abrange interações com contato físico, incluindo desde a prática de carícias, manipulação de genitais, mama ou ânus, até interações sem contato físico, como voyeurismo e exibicionismo. Estimativas sugerem que $20 \%$ das mulheres já sofreram ASI em algum momento da infância (World Health Organization, 2014) e, devido aos prejuízos para a saúde, a World Health Organization (1999) o definiu como um problema de saúde pública.

O contexto de ocorrência do ASI é considerado um dos mediadores das consequências dessa experiência na vida adulta (Borges \& Dell'Aglio, 2008). A literatura indica dois contextos de ocorrência, o intrafamiliar e o extrafamiliar (Hohendorff, Habigzang \& Koller, 2014). Existem outros fatores que podem mediar as consequências do ASI, como a duração do abuso, frequência, grau de relacionamento com o autor do abuso, idade da vítima e presença ou não de figuras protetoras que demonstrem credibilidade na vítima (Easton, Coohey, O'leary, Zhang, \& Hua, 2011).

Outros dois fatores que influenciam as consequências do abuso sexual são revitimização e polivitimização (Finkelhor, Turner, Hamby, \& Ormrod, 2011). Entende-se por revitimização novas experiências abusivas em momento diferente da vida. Por outro lado, a polivitimização é compreendida como vivências de múltiplas formas de violência (abuso sexual, negligência, abuso emocional e abuso físico), concomitantes ou em

322 diferentes momentos do ciclo vital (Finkelhor et al.,
2011). A polivitimização na infância pode aumentar as chances da vítima experienciar novos eventos traumáticos ao longo da vida (Widom, Czaja, \& Dutton, 2008). Essas vítimas estariam também mais propensas a apresentar desregulação emocional na idade adulta (Gratz, Bornovalova, Delaney-Brumsey, Nick, \& Lejuez, 2007).

A desregulação emocional pode ser compreendida como a dificuldade para lidar com as emoções, expressando-as com intensidade excessiva (i.e. agressividade) ou com diminuição excessiva (e.g. entorpecimento emocional) (Leahy, Tirch, \& Napolitano, 2013). Mulheres com histórico de trauma precoce crônico ou que sofreram revitimização de abuso sexual em algum momento da vida possuem maior tendência a apresentar dificuldade de regulação emocional, quando comparadas a outras que foram vítimas uma vez ou que não foram vítimas (Dvir, Ford, Hill, \& Frazier, 2014; Walsh, DiLillo \& Scalora, 2011). As emoções mais comumente relatadas pelas vítimas de ASI dizem respeito a nojo e tristeza, e essas emoções, somadas a estratégias de regulação emocional desadaptativas, podem contribuir para o desencadeamento de psicopatologias na idade adulta (Coyle, Karatzias, Summers, \& Power, 2014).

Vítimas de ASI também podem apresentar dificuldades nas relações sexuais na idade adulta (Castellini et al., 2013), como insatisfação sexual, pensamentos indesejados (e.g. nojo ao ser tocada), revitimização (Reid \& Sullivan, 2009), comportamentos hipersexualizados e aversão sexual (Aaron, 2012). Algumas divergências são encontradas na literatura científica em relação às dificuldades nas relações sexuais. Embora aversão sexual e disfunções sexuais sejam citadas como consequências comuns (Aaron, 2012), há evidências de aumento do de- 
sejo sexual diádico, autoerótico e excitação em vítimas de $\mathrm{ASI}$, relacionados à frequência elevada de episódios de abuso na infância (Moyano \& Sierra, 2014). Além disso, pode haver aumento de interesse sexual e menor constrangimento em relações de intimidade (Niehaus, Jackson, \& Davies, 2010). Crianças vitimizadas em idade pré-escolar tendem a apresentar comportamentos hipersexualizados, enquanto vítimas na idade escolar ou adolescência estão mais propensas à aversão sexual e também ao desenvolvimento de sentimentos de culpa (Aaron, 2012).

Pesquisas mostram que o abuso sexual vivenciado na infância pode gerar problemas nos relacionamentos amorosos na idade adulta (Lassri, Luyten, Fonagy, \& Shahar, 2018; Meyer, Cohn, Robinson, Muse, \& Hughes, 2017; Frías, Brassar, \& Shaver, 2014; Lindert et al., 2014). Os principais achados dizem respeito a vínculos afetivos e de confiança prejudicados (Frías et al., 2014), autocrítica em excesso e insatisfação nas relações amorosas (Lassri et al., 2018).

Outro desfecho do ASI é o comportamento sexual de risco. Esse comportamento é considerado arriscado quando a pessoa não faz uso de preservativo para se proteger da contaminação de doenças sexualmente transmissíveis (Ntaganira, Hass, Hosner, Brown, \& Mock, 2012). O estudo de Conley e Garza (2011) demonstrou que, quanto maior a severidade do ASI, maiores foram os níveis de comportamento sexual de risco na idade adulta.

As divergências na literatura a respeito das consequências do ASI para as vítimas durante o ciclo vital dizem respeito à complexidade desse fenômeno. A interação entre diversas variáveis (e.g. tipo de ASI, idade da vítima, rede de apoio, proximidade com o autor do abuso) pode influenciar diferentes desfechos (i.e. apresentar ou não insatisfação sexual). Diante disso, o objetivo deste estudo foi avaliar mulheres que sofreram ASI para investigar questões relacionadas à regulação emocional, à satisfação sexual e ao comportamento sexual de risco. A combinação de medidas de autorrelato e entrevista em profundidade foi utilizada para compreender as experiências de ASI e suas repercussões na idade adulta.

\section{Método}

\section{Delineamento}

Trata-se de estudo misto de caráter exploratório e descritivo.

\section{Participantes}

Participaram do estudo oito mulheres maiores de 18 anos, vítimas de ASI. A média de idade das participantes foi de 38 anos $(D P=12,7)$. O critério de inclusão foi ter sofrido pelo menos um episódio de abuso sexual até os 12 anos de idade.

\section{Instrumentos}

A pesquisa utilizou-se dos seguintes instrumentos:

(1) Questionário Sociodemográfico: Foi utilizado para caracterização sociodemográfica das participantes (idade, raça, profissão, estado civil, escolaridade).

(2) Escala de Dificuldades de Regulação Emocional (DERS): Foi utilizada para avaliação dos níveis de dificuldade de regulação emocional em seis domínios: não aceitação das emoções negativas; incapacidade de se envolver em comportamentos dirigidos por objetivos quando experiência emoções negativas; dificuldades em controlar comportamento impulsivo quando experiencia emoções negativas; acesso limitado a estratégias de regulação emocional que são perce-bidas como efetivas; falta de consciência emocional; e falta de clareza emocional. A Escala é composta por 36 itens, graduados de 1 a 5 (1: nunca, 5: sempre). O instrumento não apresenta ponto de corte; portanto, foi utilizada a média geral para essa métrica, sendo que quanto maior a pontuação, maior nível de desregulação emocional. A Escala foi desenvolvida por Gratz e Roemer (2004) e validada para o português lusitano por Coutinho, Ribeiro, Ferreirinha e Dias (2010). Apresentou Alfa de Cronbach 0,93.

(3) Questionário sobre Traumas na Infância (CTQ): Foi utilizado para investigação de cinco 
situações traumáticas: abuso físico, abuso emocional, abuso sexual, negligência física e negligência emocional. É composto por 28 itens com escala de 1 a 5 (1: nunca, 5: sempre). O questionário possui quatro pontos de corte - mínimo, moderado, grave e extremo - de acordo com o somatório das respostas para cada situação traumática. Esse instrumento é uma versão traduzida para o português por Grassi-Oliveira, Stein e Pezzi (2006), a partir do Childhood Trauma Questionnaire (CTQ), desenvolvido por Bernstein, Ahluvalia, Pogge e Handelsman (1997). Apresentou Alfa de Cronbach 0,92 .

(4) Medida Global de Satisfação Sexual (GMSEX): Foi utilizada para avaliação da satisfação sexual global no contexto de relação íntima. É composta por cinco itens relacionados à vida sexual que são respondidos por meio de uma escala de 1 a 7. O escore máximo a ser alcançado equivale a 35 pontos, e o mínimo a cinco. Como o instrumento não apresenta ponto de corte, foi utilizada a média geral para essa métrica, levando em consideração que, quanto maior o escore geral, maior a satisfação sexual. Esse instrumento foi criado por Lawrance e Byers (1995) e validado para o português lusitano por Pascoal, Narciso, Pereira e Ferreira (2013). Apresentou Alfa de Cronbach de 0,93.

(5) Questionário de Prevalência de Abuso Sexual no Brasil: Foi utilizado para acessar o tipo do abuso sexual ocorrido, duração, identificação dos autores, revelação, medidas de proteção, se o abuso cessou ou continuou após a revelação e o impacto psicológico e/ou físico. Foi desenvolvido pelo Grupo de Trabalho da Associação Nacional de Pesquisa e Pós-Graduação em Psicologia (ANPEPP) Tecnologia Social e Inovação: Intervenções Psicológicas e Práticas Forenses Contra a Violência.

(6) Entrevista Semiestruturada: Foi utilizada para compreender e aprofundar aspectos investigados nos instrumentos descritos anteriormente. As perguntas do roteiro foram referentes às relações familiares (percepção das relações familiares na infância e idade adulta), regulação emocional (descrição de situações que despertaram medo, felicidade, raiva, nojo e tristeza e como lidou com essas

324 emoções) e sexualidade (frequência das relações sexuais, sensações de prazer, uso de preservativo e atividade sexual forçada na idade adulta). O roteiro foi elaborado pelas autoras deste estudo.

\section{Procedimentos}

O presente estudo foi realizado de acordo com a Resolução 466/12 (Ministério da Saúde, 2012). O projeto foi avaliado e aprovado pelo Comitê de Ética em Pesquisa da Universidade Pontifícia Universidade Católica do Rio Grande do Sul, sob o n० 1.035.497.

O recrutamento das participantes aconteceu por meio de encaminhamentos realizados por serviços que constituem a rede de atendimento e apoio a mulheres em situação de violência (Centro de Referência da Mulher [CRM]) de Porto Alegre e região metropolitana. Os serviços que se dispuseram a participar selecionaram, dentre suas usuárias, aquelas que haviam relatado histórico de abuso sexual na infância. Todas as mulheres selecionadas foram contatadas pelos serviços, e as que demonstraram interesse em participar da pesquisa foram convidadas para entrevista com a equipe. Nesse processo identificaram-se dificuldades para contatá-las por telefone, dificuldades para agendamento da coleta em função das atividades laborais das mulheres, além do fato de que algumas não demonstraram interesse em participar da pesquisa. Em razão disso, uma nova estratégia para recrutamento de participantes foi estabelecida, e a pesquisa foi divulgada em redes sociais. Nessa divulgação foi disponibilizado o e-mail da primeira autora para contato. Algumas mulheres entraram em contato e concordaram em participar. Assim, quatro participantes eram usuárias dos serviços parceiros e quatro foram selecionadas por meio da divulgação em redes sociais.

A coleta de dados ocorreu no Serviço de Atendimento em Psicologia da Universidade, no Centro de Referência de Assistência Social, e também no Centro de Referência da Mulher. A aplicação dos instrumentos e a realização da entrevista ocorreram em um encontro com cada participante. A duração foi de aproximadamente 
duas horas. A coleta de dados ocorreu na seguinte ordem: (1) aplicação do questionário sociodemográfico; (2) aplicação em ordem aleatória das escalas de regulação emocional, satisfação sexual, traumas na infância e questionário sobre situações envolvendo o abuso; (3) aplicação da entrevista semiestruturada. As entrevistas foram gravadas e transcritas posteriormente.

\section{Análise de Dados}

Foram realizadas análises descritivas dos dados advindos dos instrumentos de autorrelato (questionário de prevalência de ASI, escalas de regulação emocional e satisfação sexual, questionário de traumas na infância) e do questionário sociodemográfico, por meio de medidas de tendência central e desvio padrão, com o uso do Statistical Package for the Social Sciences (SPSS Inc., Chicago, Illinóis, Estados Unidos) versão 20.0.

A análise das entrevistas foi orientada pelo método de análise de conteúdo temática para composição de um eixo geral de temas. Conforme Castro, Abs e Sarriera (2011), é fundamental, antes da análise dos dados qualitativos, a definição da unidade de análise e organização do material, o que é denominado pré-análise. Nessa etapa definiram-se, como unidade de análise do texto, conjuntos de frases que expressassem uma temática específica. Em seguida, iniciou-se o processo de análise, por meio da leitura exaustiva do texto e posterior aplicação de temas previamente eleitos como critérios de observação do material (inferência dedutiva). Relações familiares, autopercepção das emoções, sexualidade e relações amorosas foram os temas previamente eleitos para compor o eixo geral temático do estudo. Após essa etapa, nova leitura de cada tema evidenciou heterogeneidade de forma e conteúdo, o que motivou a criação de subtemas para maior refinamento da análise. Essa etapa do processo aderiu a uma lógica indutiva de caracterização dos subtemas, pois se baseou na identificação de diferenças de conteúdo a partir do próprio texto analisado. A última etapa da análise buscou integrar dados qualitativos, quantitativos e resultados de estudos anteriores sobre o fenômeno
(Braun \& Clarke, 2006). Como forma de aumentar a fidedignidade do crivo de análise na composição das temáticas gerais, solicitou-se a um juiz independente a aplicação do eixo geral temático ao material. As análises feitas foram posteriormente comparadas para observar eventuais discrepâncias das análises dedutivas e ajuste dos temas e subtemas.

\section{Resultados}

A idade média das participantes no momento da primeira situação de abuso sexual foi de 5,9 anos $(D P=1,8)$. Todas as participantes relataram terem sido vítimas de somente um autor, sendo cinco vítimas de abuso sexual intrafamiliar e três de abuso sexual extrafamiliar. Todos os autores do abuso eram do sexo masculino. A média de idade deles era de 35,2 anos $(D P=21,5)$. Segundo as participantes, nenhum dos autores do abuso sexual respondeu criminalmente por essa violência. A Tabela 1 apresenta informações relativas ao histórico de ASI.

Todas as participantes identificaram consequências negativas relacionadas à experiência de abuso sexual. As consequências elencadas com maior frequência a partir do "Questionário de Prevalência de Abuso Sexual no Brasil" foram: vergonha, tristeza, confusão e medo $(n=8)$, seguidos de nojo e pensamentos repetitivos sobre o evento $(n=7)$, baixo desempenho para realizar tarefas, interesse sexual precoce e pouco interesse sexual na idade adulta ( $n=6)$, raiva, pesadelos, evitação de contatos físicos com outras pessoas $(n=5)$ e, por último, vingança $(n=4)$.

Em relação às experiências de maus-tratos na infância, os resultados variaram de mínimos a extremos: violência psicológica $(M=18,1$, $D P=6,5)$; violência física $(M=13,6, D P=6,4)$; violência sexual $(M=13,7, D P=6,4)$; negligência emocional $(M=12,0, D P=6,5)$; e negligência física $(M=12,4, D P=2,2)$. Os escores de satisfação sexual apresentaram $M=21,4(D P=10,4)$, e os de regulação emocional $M=108,2(D P=20,4)$, sendo que, quanto maior o escore, maior a presença de satisfação sexual e dificuldades de regulação emocional (Tabela 2). 
Tabela 1

Histórico do abuso sexual na infância

\begin{tabular}{|c|c|c|c|c|c|c|}
\hline$P$ & Idade da vítima & Idade do autor do abuso & Vínculo do autor do abuso & Duração/Frequência & Revelação & Credibilidade \\
\hline I & 5 & 70 & Avô & 1 episódio & Não & - \\
\hline ॥ & 6 & 14 & Amigo & 1 episódio & $\operatorname{sim}$ & Não \\
\hline III & 7 & 15 & Primo & 2 anos (eventualmente) & Sim & Não \\
\hline IV & 3 & 60 & Tio & 9 anos (diariamente) & Sim & Não \\
\hline V & 5 & 15 & Irmão & 5 anos (eventualmente) & Não & - \\
\hline $\mathrm{VI}$ & 5 & 28 & Amigo do pai & 1 episódio & Sim & Não \\
\hline VII & 7 & 35 & Vizinho & 4 anos (eventualmente) & Sim & Não \\
\hline VIII & 9 & 45 & Tio & 1 episódio & Sim & Não \\
\hline
\end{tabular}

Nota: P: Participantes.

Tabela 2

Niveis de maus-tratos, indices de satisfação sexual e regulação emocional

\begin{tabular}{|c|c|c|c|c|c|c|c|c|c|c|c|c|}
\hline \multirow{2}{*}{$\begin{array}{l}\mathrm{P} \\
\\
\text { I }\end{array}$} & \multicolumn{2}{|c|}{$\begin{array}{l}\text { Violência } \\
\text { Psicológica } \\
\text { (Escore) }\end{array}$} & \multicolumn{2}{|c|}{$\begin{array}{l}\text { Violência } \\
\text { Física } \\
\text { (Escore) }\end{array}$} & \multicolumn{2}{|c|}{$\begin{array}{l}\text { Violência } \\
\text { Sexual } \\
\text { (Escore) }\end{array}$} & \multicolumn{2}{|c|}{$\begin{array}{c}\text { Negligência } \\
\text { Emocional } \\
\text { (Escore) }\end{array}$} & \multicolumn{2}{|c|}{$\begin{array}{c}\text { Negligência } \\
\text { Física } \\
\text { (Escore) }\end{array}$} & \multirow{2}{*}{$\begin{array}{c}\text { Índice de } \\
\text { Satisfação } \\
\text { Sexual }\end{array}$} & \multirow{2}{*}{$\begin{array}{c}\text { Índice de } \\
\text { Desregulação } \\
\text { Emocional }\end{array}$} \\
\hline & Extremo & $(25)$ & Extremo & (20) & Grave & $(12)$ & Mínimo & (9) & \multicolumn{2}{|c|}{ Moderado(12) } & & \\
\hline$\|$ & Moderad & $\mathrm{o}(12)$ & Mínimo & (7) & Mínimo & (5) & Extremo & (19) & \multicolumn{2}{|c|}{ Moderado(12) } & 31 & 117 \\
\hline III & Extremo & (17) & Extremo & (17) & Grave & $(12)$ & \multicolumn{2}{|c|}{ Moderado(10) } & \multicolumn{2}{|c|}{ Moderado(12) } & 26 & 123 \\
\hline IV & Extremo & (23) & Extremo & (15) & Extremo & (20) & Mínimo & (6) & Mínimo & (9) & 5 & 137 \\
\hline V & Extremo & $(22)$ & Grave & $(12)$ & Extremo & (25) & Moderac & $o(10)$ & Extremo & (17) & 30 & 100 \\
\hline VI & Mínimo & (6) & Mínimo & (6) & Grave & (8) & Mínimo & (5) & Mínimo & (5) & 29 & 82 \\
\hline VII & Extremo & (23) & Extremo & (15) & Extremo & $(16)$ & Mínimo & (7) & Grave & $(12)$ & 17 & 80 \\
\hline VIII & Extremo & (17) & Extremo & $(17)$ & Grave & $(12)$ & Moderac & $\mathrm{o}(10)$ & Grave & $(12)$ & 26 & 123 \\
\hline
\end{tabular}

Nota: P: Participantes.

Por fim, foi constatado que todas as participantes haviam realizado psicoterapia em algum momento de suas vidas. No momento da coleta, três estavam em acompanhamento psicoterapêutico (participantes I, II e IV). A duração da psicoterapia variou de um a seis anos.

Em relação à análise de conteúdo, além dos eixos temáticos definidos a priori, identificou-se "Percepção sobre a terapia" como um tema emergente. Desses temas, identificou-se a necessidade

326 de criar subtemas, com o objetivo de atender às especificidades do conteúdo. A Tabela 3 apresenta os temas, subtemas e unidades representativas de análise, identificados a partir das entrevistas.

\section{Discussão}

Identificou-se que os episódios de abuso iniciaram em idade pré-escolar. O contexto foi predominantemente intrafamiliar e pelo menos a metade das participantes relataram terem sido vítimas de ASI mais de uma vez, com exposição 
Tabela 3

Temas, subtemas e unidades de análise representativas

\begin{tabular}{|c|c|c|}
\hline Temas & Subtemas & Unidade de Análise Representativa \\
\hline \multirow{4}{*}{$\begin{array}{l}\text { Relações } \\
\text { Familiares }\end{array}$} & Desamparo & $\begin{array}{l}\text { "... eu fiquei gravida ne, com } 12 \text { anos, do abuso, aí minha mãe e minha tia me colocaram num } \\
\text { quarto ... abriram as minhas perna e colocaram o caninho lá dentro, eu já tava de barriga ne, ... e } \\
\text { quando vê o nenê tava saindo, nas mãos delas ... tinha cabelinho, mãozinha, pezinho ... tava vivo } \\
\text { ainda e eu pedi pra elas não fazerem nada ... colocaram numa caixinha de papelão, enrolaram num } \\
\text { pano e enterraram embaixo de um pé de caqui ..." (p.IV). }\end{array}$ \\
\hline & $\begin{array}{l}\text { Abuso físico, } \\
\text { emocional e sexual }\end{array}$ & $\begin{array}{l}\text { "... minha mãe sofria violência doméstica, ... eu e minha irmã sofríamos violência sexual, então } \\
\text { minha mãe sempre queria fugir de casa. Ela sempre queria me levar e eu não queria deixar meus } \\
\text { irmãos, então eu acabava voltando e sofrendo a violência de novo" (p.III). }\end{array}$ \\
\hline & $\begin{array}{l}\text { Ambivalência (afeto } \\
\text { e hostilidade) }\end{array}$ & $\begin{array}{l}\text { "... eu lembro de ter uns sete anos e ter feito alguma coisa errada com as minhas figurinhas ... e ele } \\
\text { me chamou de cadela ..., mas ele sempre foi muito afetuoso, então é uma coisa bem confusa ..." (p.II). }\end{array}$ \\
\hline & $\begin{array}{l}\text { Falta de } \\
\text { proximidade afetiva }\end{array}$ & $\begin{array}{l}\text { "... quando eu fiquei menstruada ... fui na minha vizinha, pra ... me ensinar como é que botava o } \\
\text { absorvente e tal. ... eu não lembro assim, em nenhum momento a minha mãe participava, assim, } \\
\text { nem o meu pai ..." (p.V). }\end{array}$ \\
\hline
\end{tabular}

\begin{tabular}{|c|c|c|}
\hline \multirow{3}{*}{$\begin{array}{l}\text { Autopercepção } \\
\text { sobre Emoções }\end{array}$} & Intensidade & $\begin{array}{l}\text { "Eu sou muito intensa ... Eu percebo que às vezes ... é uma onda de emoção muito forte, que } \\
\text { demora pra passar e tal" (p.II). }\end{array}$ \\
\hline & $\begin{array}{l}\text { Descontrole } \\
\text { emocional }\end{array}$ & $\begin{array}{l}\text { "... tem uma fase que eu queria ferir todo mundo ... aí, eu fico muito mal assim quando eu estou } \\
\text { com raiva ..." (p.III). } \\
\text { "... eu coloquei fogo na minha casa, com meu ex marido dentro ... eu coloquei veneno de rato na } \\
\text { comida dele e esperei ele comer" (p.IV). }\end{array}$ \\
\hline & $\begin{array}{c}\text { Dificuldade de } \\
\text { manejo das } \\
\text { emoções }\end{array}$ & $\begin{array}{l}\text { "... é que eu falo coisas, assim, que não precisaria, mas é pra me defender, é pra atingir. Porque é } \\
\text { uma forma de "eu tô sofrendo e tu vai sofrer também" sabe?" (p.V). } \\
\text { "... se eu brigar contigo eu vou te falar horrores, vou ferrar contigo, durante cinco minutos, e aí } \\
\text { depois a gente pode ir tomar um sorvete juntas, entendeu?" (p.II). }\end{array}$ \\
\hline
\end{tabular}

"... sempre teve essa cobrança assim, porque eu nunca gostei de sexo ... hoje eu gosto (se referindo Satisfação sexual ao relacionamento extraconjugal) ... eu descobri a minha sexualidade ne, eu descobri um prazer que eu não sentia antes" (p.III).

\begin{tabular}{|c|c|c|}
\hline \multirow{5}{*}{ Sexualidade } & . & $\begin{array}{l}\text { ao relacıonamento extraconjugal) ... eu descobrı a minha sexualıdade ne, eu descobrı um praze } \\
\text { que eu não sentia antes" (p.III). }\end{array}$ \\
\hline & $\begin{array}{l}\text { Desejo sexual } \\
\text { precoce }\end{array}$ & $\begin{array}{l}\text { "... não tinha mais como eu fugir ... então eu acabava participando, acho que despertou a minha } \\
\text { sexualidade ..." (p.III). }\end{array}$ \\
\hline & $\begin{array}{l}\text { Comportamento } \\
\text { hipersexualizado }\end{array}$ & $\begin{array}{l}\text { "... eu sou uma pessoa que transa bastante ... eu sou uma pessoa bem sexual ... todo mundo me } \\
\text { tem como uma guru do sexo, entendeu (p.II). }\end{array}$ \\
\hline & Aversão sexual & $\begin{array}{l}\text { "Uns quatro anos que eu não tenho relação, eu não consigo ne ... nunca senti prazer ... nunca senti } \\
\text { vontade ... sempre foi uma violência" (p.IV). }\end{array}$ \\
\hline & $\begin{array}{l}\text { Comportamento } \\
\text { sexual de risco }\end{array}$ & $\begin{array}{l}\text { "Me coloquei em risco várias vezes ... Acho também que eu tinha uma tendência de achar que a } \\
\text { pessoa é uma pessoa muito bacana dai não precisa usar camisinha ..." (p. II). }\end{array}$ \\
\hline \multirow{4}{*}{$\begin{array}{l}\text { Relações } \\
\text { Amorosas }\end{array}$} & $\begin{array}{l}\text { Impulsividade } \\
\text { no manejo das } \\
\text { relações amorosas }\end{array}$ & $\begin{array}{l}\text { "... os meus relacionamentos começam bem rápido, ou rola conexão ou não rola ... todos os meus } \\
\text { relacionamentos eu estava saindo um mês com a pessoa e comecei a namorar" (p.II). }\end{array}$ \\
\hline & Relações conflitivas & "É uma relação de conflitos ... a gente ta vinte anos juntos, mas sempre teve conflitos. ... (p.III). \\
\hline & $\begin{array}{l}\text { Percepção } \\
\text { positiva dos } \\
\text { relacionamentos }\end{array}$ & $\begin{array}{l}\text { "Meu relacionamento hoje é um relacionamento pleno assim ... eu e ele somos bem companheiros } \\
\text {... pensamos em construir algo juntos sabe" (p.VIII). }\end{array}$ \\
\hline & $\begin{array}{l}\text { Medo de } \\
\text { revivenciar abuso } \\
\text { na idade adulta }\end{array}$ & $\begin{array}{l}\text { "... se tu é vulnerável, tu é abusado, ou tu é abusado psicologicamente, ou tu é abusado } \\
\text { emocionalmente, ou se aproveitam de ti por causa de dinheiro, sabe .... Eu tô sempre me defendendo } \\
\text { porque eu sei que alguém vai abusar de mim de alguma forma ..." (p.V). }\end{array}$ \\
\hline $\begin{array}{l}\text { Percepção sobre } \\
\text { a Psicoterapia }\end{array}$ & $\begin{array}{l}\text { Benefícios da } \\
\text { psicoterapia }\end{array}$ & $\begin{array}{l}\text { "porque no passado eu me afastava, depois com o tratamento, a terapia, enfim. Hoje já é bem } \\
\text { diferente" (p.I). } \\
\text { "Já fiz duas terapias ... melhorei bastante em muitos quesitos, porque eu era violenta ... mas a } \\
\text { psicóloga me fez entender que isso era uma reprodução da infância, então eu aboli bater nas minhas } \\
\text { filhas ... acho que se eu não tivesse feito terapia eu não teria chegado até aqui" (p.III). }\end{array}$ \\
\hline
\end{tabular}


prolongada. Os níveis de abuso sexual foram graves ou extremos na maioria dos casos. Além disso, todas as participantes indicaram experienciar outras formas de maus-tratos na infância, como abusos emocionais, físicos e negligência, caracterizando assim a polivitimização. A literatura aponta que vivenciar um episódio abusivo/traumático pode facilitar a ocorrência de novas experiências nesses contextos (Finkelhor, Turner, Shattuck, \& Hamby, 2015).

Em relação à revelação da ocorrência de abuso sexual, as participantes que o fizeram foram vistas com descrédito pelas pessoas que deveriam zelar pelo seu bem-estar, inviabilizando assim que medidas protetivas fossem tomadas. Sabe-se que o descrédito no relato das crianças sobre o abuso sexual é frequente pelos adultos (Santos \& Costa, 2011).

Os resultados da escala de desregulação emocional indicaram que metade das participantes pontuaram índices acima da média nessa amostra. Já na entrevista, todas as participantes relataram situações de desregulação emocional envolvendo alta intensidade de expressão, dificuldades no manejo e episódios nos quais sentiram descontrole emocional. As participantes que foram vítimas de violência intrafamiliar, com recorrência de episódios de abuso por alguém que deveria exercer o papel protetivo, demonstraram níveis mais elevados de desregulação emocional. A literatura corrobora esses achados, uma vez que um dos fatores mediadores da desregulação emocional está relacionado ao fato de o autor da violência exercer o papel de figura protetora da criança (Cloitre, Miranda, Stovall-McClough, \& Han, 2005). Além disso, mulheres vítimas de ASI que sofreram polivitimização podem apresentar maior dificuldade para regular suas emoções do que aquelas que sofreram somente um ou nenhum episódio de abuso (Walsh et al., 2011).

Os resultados obtidos por meio da escala de satisfação sexual indicaram que mais da metade das participantes apresentaram escore acima da média, revelando níveis positivos de satisfação sexual. No entanto, o conteúdo das entrevistas indicou comportamento hipersexualizado. Estudos prévios também evidenciam não haver consenso em relação às consequências no âmbito da sexualidade (Aaron, 2012; Moyano \& Sierra, 2014). Esse resultado também aponta a importância de combinar diferentes estratégias de coleta de dados para compreender um fenômeno. A escala não permitiu identificar conteúdos expressos nas entrevistas.

A aversão sexual, evidenciada, por exemplo, no relato da participante IV pode estar relacionada à polivitimização (e.g. abuso físico, psicológico, sexual) em nível extremo, cronicidade do abuso e também ao vínculo com o autor do abuso. As emoções, principalmente o nojo, responsável por evocar situações de evitação, também podem estar associadas a dificuldades nas relações sexuais (Turchik, 2012).

Em relação ao comportamento hipersexualizado, conforme é evidenciado no relato da participante II, pode ser compreendido pela ocorrência única de abuso sexual, em idade precoce, conforme resultados indicados pela pesquisa de Aaron (2012). Além disso, aspectos como contexto extrafamiliar, autor do abuso com faixa etária próxima à da vítima podem ser atenuantes nas consequências ao longo do desenvolvimento.

O despertar precoce da sexualidade também se mostrou um desfecho nessa pesquisa. Acredita-se que situações abusivas recorrentes, principalmente em idade precoce, podem estar associadas a esse resultado. Vivenciar situações recorrentes de abuso sexual na infância pode despertar precocemente o desejo sexual (Moyano \& Sierra, 2014).

Por fim, as participantes que relataram apenas um episódio de ASI e apresentaram índices mínimos a moderados de maus-tratos na infância, pontuaram níveis de satisfação sexual elevados na escala de autorrelato. A literatura aponta que sofrer episódio único de abuso sexual na infância pode não afetar significativamente o funcionamento sexual em comparação a vítimas recorrentes (Moyano \& Sierra, 2014).

No que se refere ao comportamento sexual de risco, duas participantes (II e VIII) relataram episódios relacionados à não utilização de preservativos nas 
relações sexuais, bem como envolvimento com parceiros abusivos. Sabe-se que comportamentos de risco, tanto relacionais (De Antoni, Yunes, Habigzang, \& Koller, 2011) quanto sexuais, também são indicados como consequências de longo prazo do ASI (Reid \& Sullivan, 2009; Fergusson, McLeod, \& Horwood, 2013). Indivíduos com histórico de violência podem apresentar dificuldades para discriminar estímulos ameaçadores ao se engajar em relacionamentos amorosos e sexuais de risco (Hansen, Brown, Tsatkin, Zelgowski \& Nightingale, 2012). O comportamento sexual é influenciado pelo ASI, no entanto, não há desfecho único em relação às consequências (Staples, Rellini, \& Roberts, 2012; Turchik, 2012; Aaron, 2012; Moyano \& Sierra, 2014; Krindges, Macedo, \& Habigzang, 2016).

Ao avaliarem suas relações amorosas, as participantes relataram situações conflitivas e de impulsividade, assim como medo da repetição de situações de abuso na idade adulta. Em decorrência de um manejo disfuncional das emoções, vítimas de ASI tendem a possuir dificuldades no âmbito relacional na vida adulta (Staples et al., 2012; Turchik, 2012). Medo e nojo, emoções frequentemente vivenciadas pelas vítimas de ASI (Frías et al., 2014; Coyle et al., 2014), também podem influenciar negativamente as relações amorosas. Isso ratifica as informações fornecidas pelas participantes, ao elencarem essas emoções como as principais consequências após a ocorrência do abuso. O medo, apesar de ser adaptativo, em diferentes circunstâncias pode ser manejado de forma disfuncional pelas vítimas de ASI, com o objetivo de evitar revitimizações. Isso pode ser observado no caso da participante $V$, que relata se defender demasiadamente em suas relações, sejam elas amorosas, familiares ou de amizade, em razão do medo de ser abusada novamente, acarretando assim a dificuldade de confiar nas pessoas.

A partir do relato das participantes identificou-se que elas vivenciaram situações familiares de invalidação (e.g. descrédito diante dos sentimentos e emoções), desamparo, abuso físico, emocional e de ambivalência (e.g. hostilidade e afeto) na sua infância e ao longo do seu desenvolvimento. Sabe-se que a família e/ou pessoas que exerçam essa função têm papel fundamental no desenvolvimento cognitivo e emocional dos indivíduos, seja para um desenvolvimento saudável ou não (World Health Organization, 2002). Crianças vítimas de violência nos primeiros anos de vida podem sofrer consequências negativas ao longo do desenvolvimento (Ministério da Saúde, 2002), principalmente se o abuso ocorrer em âmbito familiar e elas não se sentirem acolhidas e protegidas em contexto de revelação (Baía, Veloso, Habigzang, Dell'Aglio, \& Magalhães, 2015).

Diante de uma experiência negativa como o ASI e das consequências relatadas pelas participantes, a terapia foi mencionada como um recurso importante, que resultou em benefícios para elas. Todas as participantes realizaram acompanhamento psicoterápico em algum momento de suas vidas e algumas ainda permanecem em acompanhamento. As participantes relataram benefícios dessa prática, principalmente no que diz respeito à regulação das emoções e estratégias de enfrentamento diante de situações adversas. Resultados de pesquisas internacionais mostram que abordagens de psicoterapia focadas no ASI têm efeitos positivos, como o ajustamento funcional das emoções. Os resultados deste estudo evidenciam a importância de investir em protocolos de tratamento efetivos para essa população.

\section{Considerações Finais}

A partir da investigação por meio de escalas de autorrelato e entrevistas, foi possível compreender que o vínculo que a vítima possui com o autor do abuso, a frequência em que o abuso ocorreu, a idade da vítima, a presença de figuras protetoras e a polivitimização foram fatores que contribuíram para minimizar ou maximizar as consequências do ASI na vida adulta. O delineamento misto possibilitou compreender aspectos da vitimização sexual na infância e como ela pode afetar a regulação das emoções e a sexualidade na idade adulta. Os resultados deste estudo apontaram que a exposição ao ASI pode gerar diferentes desfechos, corroborando a literatura da área. Fatores individuais, da rede de proteção e da violência em si contribuem para que 
vítimas apresentem diferentes consequências ao longo do desenvolvimento.

Esta pesquisa apresentou algumas limitações. O reduzido número de participantes impossibilitou análises estatísticas inferenciais entre as variáveis investigadas. Por outro lado, não comprometeu a perspectiva exploratória proposta pelo estudo. As pesquisadoras encontraram dificuldade de acesso às participantes. Essa dificuldade gerou a necessidade de divulgar a pesquisa em redes sociais. O recrutamento de participantes por meio de redes sociais pode representar outra limitação, pois há duas formas distintas de seleção da amostra. Por meio das redes sociais pode-se ter acessado mulheres com maior disponibilidade para compartilhar experiências negativas como o abuso sexual.

Outra limitação refere-se aos procedimentos de coleta dos dados. Os instrumentos de autorrelato e a entrevista foram coletados no mesmo encontro, de modo que as respostas aos itens dos instrumentos podem ter influenciado as respostas à entrevista. Por outro lado, a entrevista permitiu aprofundar aspectos experienciais das participantes. Por fim, o tempo de coleta pode ter representado um desgaste para as participantes. Embora elas tivessem sido orientadas a interromper a coleta caso necessitassem de um intervalo ou de outro dia para finalização, a fim de evitar possíveis impactos negativos do tempo de coleta, nenhuma delas solicitou tal interrupção.

Apesar dessas limitações, os resultados encontrados poderão subsidiar intervenções psicossociais para mulheres com histórico de ASI. Verifica-se a necessidade de considerar nas intervenções aspectos relacionados à regulação emocional e à sexualidade. Foram identificadas no cenário nacional escassas pesquisas empíricas que tratem das consequências desenvolvimentais do ASI em longo prazo. Assim como orientações para intervenções com essa população adulta. Estudos futuros podem investigar o impacto da psicoterapia na regulação emocional, satisfação sexual e comportamento sexual de risco, por meio de comparação entre grupos de mulheres com diferentes históricos de ASI, por exemplo.

\section{Colaboradores}

C.A. KRINDGES foi responsável pelo desenho, coleta de dados, análise dos dados, elaboração da discussão, resultados e aprovação da versão final. L.F. HABIGZANG, orientadora responsável pela pesquisa, participou da formulação do problema, desenho, revisão e aprovação da versão final.

\section{Referências}

Aaron, M. (2012). The pathways of problematic sexual behavior: A literature review of factors affecting adult sexual behavior in survivors of childhood sexual abuse. Sexual Addiction and Compulsivity, 19, 199-218. http://dx.doi.org/10.1080/10720162.2012.690678

Baía, P. A. D., Veloso, M. M. X., Habigzang, L. F., Dell'Aglio, D. D., \& Magalhães, C. M. C. (2015). Padrões de revelação e descoberta do abuso sexual de crianças e adolescentes. Revista de Psicologia, 24(1), 1-19. http://dx.doi/ 10.5354/0719-0581.2015.37007

Bernstein, D. P., Ahluvalia, T., Pogge, D., \& Handelsman, L. (1997). Validity of the childhood trauma questionnaire in an adolescent psychiatric population. Journal of the American Academy of Child and Adolescent Psychiatry, 36(3), 340-348.

Braun, V., \& Clarke, V. (2006) Using thematic analysis in psychology. Qualitative Research in Psychology, 3(2), 77-101. http://dx.doi.org/10.1191/1478088706qp063oa

Borges, J. L., \& Dell'Aglio, D. D. (2008). Relações entre abuso sexual na infância, transtorno de estresse pós-traumático (TEPT) e prejuízos cognitivos. Psicologia em Estudo, 13(2), 371-379.

Castellini, G., Sauro, C. L., Lelli, L., Godini, L., Vignozzi, L., Rellini, A. H. ... Ricca, V. (2013). Childhood sexual abuse moderates the relationship between sexual functioning and eating disorder psychopathology in anorexia nervosa and bulimia nervosa: A 1-year followup study. Journal of Sexual Medicine, 10(9), 2190-2200. http://dx.doi.org/10.1007/s40519-015-0195-y

Castro, T. G., Abs, D., \& Sarriera, J. C. (2011). Análise de conteúdo em pesquisas de Psicologia. Psicologia: Ciência e Profissão, 31(4), 814-825.

Cloitre, M., Miranda, R., Stovall-McClough, K. C., \& Han, H. (2005). Beyond PTSD: Emotion regulation and interpersonal problems as predictors of functional impairment in survivors of childhood abuse. Behavior Therapy, 36(2), 119-124.

Conley, T. D., \& Garza, M. R. (2011). Gender and sequelae of child versus adult onset of sexual victimization: Body mass, binge eating, and promiscuity. Journal of Applied Social Psychology, 41(11), 2551-2572. http:// dx.doi.org/10.1111/j.1559-1816.2011.00828.x 
Coutinho, J., Ribeiro, E., Ferreirinha, R., \& Dias, P. (2010). Versão portuguesa da escala de dificuldades de regulação emocional e sua relação com sintomas psicopatológicos. Revista de Psiquiatria Clínica, 37(4), 145-151. http://dx.doi.org/10.1590/S0101-60 832010000400001

Coyle, E., Karatzias, T., Summers, A., \& Power, M. (2014). Emotions and emotion regulation in survivors of childhood sexual abuse: The importance of "disgust" in traumatic stress and psychopathology. European Journal of Psychotraumatology 5(1). http://dx.doi. org/10.3402/ejpt.v5.23306

De Antoni, C., Yunes, M. A. M., Habigzang, L. F, \& Koller, S. H. (2011). Abuso sexual extrafamiliar: percepções das mães de vítimas. Estudos de Psicologia (Campinas), 28(1), 97-106. http://dx.doi.org/10.1590/S0103-16 6X2011000100010

Dvir, Y., Ford, J. D., Hill, M., \& Frazier, J. A. (2014). Childhood maltreatment, emotional dysregulation, and psychiatric comorbidities. Harvard Review of Psychiatry, 22(3), 149-161. http://dx.doi.org/10.1097/ HRP.0000000000000014

Easton, S. D., Coohey, C., O'leary, P., Zhang, Y., \& Hua, L. (2011). The effect of childhood sexual abuse on psychosexual functioning during adulthood. Journal of Family Violence, 26(1), 41-50. http://dx.doi. org/10.1007/s10896-010-9340-6

Fergusson, D. M., McLeod, G. F., \& Horwood, L. J. (2013). Childhood sexual abuse and adult developmental outcomes: Findings from a 30-year longitudinal study in New Zealand. Child Abuse \& Neglect, 37(9), 664-74. http://dx.doi.org/10.1016/j.chiabu.2013.03.013

Finkelhor, D., Turner, H., Shattuck, A., \& Hamby, S. (2015). Prevalence of childhood exposure to violence, crime, and abuse. JAMA Pediatrics, 169(8), 746-754. http:// dx.doi.org/10.1001/jamapediatrics.2015.0676

Finkelhor, D., Turner, H., Hamby, S., \& Ormrod, R. (2011). Polyvictimization: Children's exposure to multiple types of violence, crime, and abuse. Juvenile Justice Bulletin NCJ 235504, 1-12.

Frías, M. T., Brassard, A., \& Shaver, P. R. (2014). Childhood sexual abuse and attachment insecurities as predictors of women's own and perceived-partner extradyadic involvement. Child Abuse \& Neglect, 38(9), 1450-1458. http://dx.doi.org/10.1016/j.chiabu.2014.02.009

Grassi-Oliveira, R., Stein, L. M., \& Pezzi, J. C. (2006). Tradução e validação de conteúdo da versão em português do Childhood Trauma Questionnaire. Revista de Saúde Pública, 40(2), 249-255.

Gratz K. L., \& Roemer L. (2004). Multidimensional assessment of emotion regulation and dysregulation: Development, factor structure, and initial validation of the Difficulties in Emotion Regulation Scale. Journal of Psychopathology and Behavioral Assessment, 26(1), 41-54. http://dx.doi.org/10.1023/B:JOBA.00 00007455.08539 .94
Gratz, K. L., Bornovalova, M. A., Delaney-Brumsey, A., Nick, B., \& Lejuez, C. W. (2007). A laboratory based study of the relationship between childhood abuse and experiential avoidance among inner-city substance abusers: The role of emotional nonacceptance. Behavior Therapy, 38(3), 256-268.

Hansen, N. B., Brown, L. J., Tsatkin, E., Zelgowski, B., \& Nightingale, V. (2012). Dissociative experiences during sexual behavior among a sample of adults living with HIV infection and a history of childhood sexual abuse. Journal of Trauma and Dissociation, 13(3), 345-360. http://dx.doi.org/10.1080/15299732.2011.641710

Hohendorff, J. V., Habigzang, L. F., \& Koller, S. H. (2014). Violência sexual contra meninos: teoria e intervenção. Curitiba: Juruá.

Krindges, C. A., Macedo, D. M., \& Habigzang, L. F. (2016). Abuso sexual na infância e suas repercussões na satisfação sexual na idade adulta de mulheres vítimas. Contextos Clínicos, 9(1), 60-71. http://dx.doi. org/10.4013/ctc.2016.91.05

Lassri, D., Luyten, P., Fonagy, P., \& Shahar, G. (2018). Undetected scars? Self-criticism, attachment, and romantic relationships among otherwise wellfunctioning childhood sexual abuse survivors. Psychological Trauma, 10(1), 121-129. http://dx.doi. org/10.1037/tra0000271

Lawrance, K. A., \& Byers, E. S. (1995). Sexual satisfaction in long-term heterosexual relationships: The interpersonal exchange model of sexual satisfaction. Personal Relationships, 2(4), 267-285.

Leahy, R. L., Tirch, D., \& Napolitano, L. A. (2013). Regulação emocional em psicoterapia: um guia para o terapeuta cognitivo-comportamental. Porto Alegre: Artmed.

Lindert, J., Ehrenstein, O. S, Grashow, R., Gal, G., Braehler, E., \& Weisskopf, M. G. (2014). Sexual and physical abuse in childhood is associated with depression and anxiety over the life course: Systematic review and meta-analysis. International Journal of Public Health (59)2, 359-372. http://dx.doi.org/10.1007/s00038-0 13-0519-5

Meyer, D., Cohn, A., Robinson, B., Muse, F., \& Hughes, R. (2017). Persistent complications of child sexual abuse: Sexually compulsive behaviors, attachment, and emotions. Journal of Child Sexual Abuse 26(2), 140-157. http://dx.doi.org/10.1007/s00038-013-0519-5

Ministério da Saúde (Brasil). (2002). Notificação de maus-tratos contra crianças e adolescentes pelos profissionais de saúde: um passo a mais na cidadania em saúde (p.14). Brasília: Ministério da Saúde.

Ministério da Saúde (Brasil). (2012). Conselho Nacional de Saúde. Resolução $n^{\circ}$ 466, de 12 de dezembro de 2012. Diretrizes e normas regulamentadoras de pesquisas envolvendo seres humanos. Recuperado em abril 13, 2014, de http://conselho.saude.gov.br/ resolucoes/2012/Reso466.pdf 
Moyano, N., \& Sierra, J. C. (2014). Funcionamiento sexual en hombres y mujeres víctimas de abuso sexual en la infancia y en La adolescencia/adultez. Revista Internacional de Andrología, 12(4), 32-38. http:// dx.doi.org/10.1016/j.androl.2014.04.012

Niehaus, A. F., Jackson, J., \& Davies, S. (2010). Sexual self-schemas of female child sexual abuse survivors: Relationships with risky sexual behavior and sexual assault in adolescence. Archives of Sexual Behavior, 39(6), 1359-1374. http://dx.doi.org/10.1007/s10508-0 10-9600-9

Ntaganira, J., Hass, L. J., Hosner, S., Brown, L., \& Mock, N. B. (2012). Sexual risk behaviors among youth heads of household in Gikongoro, south province of Rwanda. BMC Public Health, (12)225. http://dx.doi. org/10.1186/1471-2458-12-225

Pascoal, P. M., Narciso, I. S. B., Pereira, N. M., \& Ferreira, A. S. (2013). Processo de validação da Global Measure of Sexual Satisfaction em três amostras da popu-lação portuguesa. Psicologia: Reflexão e Crítica, 26(4), 691-700. http://dx.doi.org/10.1590/S0102-7972 2013000400009

Reid, J. A., \& Sullivan, C. J. (2009). A model of vulnerability for adult sexual victimization: The impact of attachment, child maltreatment, and scarred sexuality. Violence and Victims, 24(4), 485-501. http:// dx.doi.org/10.1891/0886-6708.24.4.485

Santos, V. A., \& Costa, L. F. (2011). A violência sexual contra crianças e adolescentes: conhecer a realidade possibilita a ação protetiva. Estudos de Psicologia (Campinas), 28(4), 529-537. http://dx.doi.org/10. 1590/S0103-166X2011000400013

Staples, J., Rellini, A. H., \& Roberts, S. P. (2012). Avoiding experiences: Sexual dysfunction in women with a history of sexual abuse in childhood and adolescence. Archives of Sexual Behavior, 41(2), 341-350. http:// dx.doi.org/10.1007/s10508-011-9773-x

Turchik, J. A. (2012). Sexual victimization among male college students: Assault severity, sexual functioning, and health risk behaviors. Psychology of Men and Masculinity, 13(3), 243-255. http://dx.doi.org/10.103 7/a0024605

Walsh, K., DiLillo, D., \& Scalora, M. (2011). The cumulative impact of sexual revictimization on emotion regulation difficulties: An examination of female inmates. Violence Against Women, 17(8), 1103-1118. http://dx.doi. org/10.1177/1077801211414165

Widom, C. S., Czaja, S. J., \& Dutton, M.A. (2008). Childhood victimization and lifetime revictimization. Child Abuse and Neglect, 32(8), 785-796. http://dx. doi.org/10.1016/j.chiabu.2007.12.006

World Health Organization. (1999). WHO recognizes child abuse as a major public health problem. The Lancet, 353(9161), 1340. https://doi.org/10.1016/S0 $140-6736(05) 74346-4$

World Health Organization. (2002). Defining sexual health: Report of a technical consultation on sexual health 28-31 January 2002. Retrieved October, 12, 2015, from http://www.who.int/reproductivehealth/ publications/sexual_health/defining_sexual_health.pdf

World Health Organization. (2014). Sexual abuse and neglect. Retrieved April 13, 2014, from http://www. who.int/violence_injury_prevention/violence/child/en/

Recebido: dezembro 21, 2016

Versão final: maio 3, 2017

Aprovado: agosto 7, 2017 